\title{
Three-dimensional simulation of Marangoni flow and interfaces in floating-zone silicon crystal growth
}

\author{
C.W. Lan*, J.H. Chian \\ Chemical Engineering Department, National Taiwan University, Taipei, Taiwan 10617, People's Republic of China
}

\begin{abstract}
Three-dimensional (3D) simulation is conducted for the floating-zone (FZ) growth of silicon in an ellipsoid mirror furnace, for the first time, simultaneously considering the time-dependent Marangoni flow, heat transfer, and moving interfaces. The numerical method is based on an efficient multigrid finite-volume method with front tracking. The growth of an $8 \mathrm{~mm}$ diameter silicon crystal under microgravity is considered. A half-zone configuration is also used for benchmarking, and the calculated bifurcation points and flow structures are in good agreement with previous results. However, quasi-periodic modes and angular waves are observed at higher Marangoni number. For full zone calculations, including the feeding and growth interfaces, the bifurcation behavior is similar, but the primary bifurcation is found to be subcritical and the 3D one-fold flow mode is dominant. Significant growth rate fluctuations and back melting are found for a typical growth condition as well. The major fluctuation frequency ranges from 0.1 to $0.3 \mathrm{~Hz}$, and the severe back melting may be related to the angular waves. (C) 2001 Elsevier Science B.V. All rights reserved.
\end{abstract}

PACS: 44.25. +f; 47.27.Te; 81.10.Fq; 02.60.c6; 02.70.Fj

Keywords: A1. Computer simulation; A1. Convection; A1. Heat transfer; A1. Interfaces; A1. Solidification; A2. Floating zone technique; A2. Growth from melt; A2. Microgravity conditions; A2. Single crystal growth

\section{Introduction}

Floating-zone (FZ) crystal growth is a popular technique for growing single crystals. Particularly, it is a crucibleless process, so that the contamination from the crucibles could be avoided. However, the free surface of the molten zone often induces significant Marangoni flow leading to unstable heat flows and distorted interfaces, even in the microgravity environment [1,2]. Severe growth striations due to the unstable flow are

*Corresponding author. Tel.: 886-2-2363-3917; fax: 886-22363-3917.

E-mail address: lan@ruby.che.ntu.edu.tw (C.W. Lan). typical, which are believed to the cause of the unstable growth rate (even with back melting). Numerical simulation provides a quick way for better understanding, especially in the interplay of the heat flows and the growth phenomena. Most of the numerical models for FZ crystal growth are two-dimensional (e.g., $[3,4]$ ), in which the heat transfer, fluid flow, and interfaces are considered. However, many experimental and numerical evidences (e.g., [5-7]) indicate that, even with a perfect growth condition, unsteady three-dimensional (3D) heat flows prevail in the molten zone. Hence, a 3D model is required and this enables the development of several 3D models (e.g., [5,6]). Unfortunately, in these models, the moving 
phase boundaries have not yet been taken into account. In fact, to model the growth in a selfconsistent manner, the moving interfaces cannot be ignored.

In this study, a self-consistent 3D model is developed. An efficient numerical algorithm using a multigrid finite volume method (FVM) with front tracking is adopted to simulate the unsteady Marangoni flow and the moving interfaces. Through this 3D model, the nonlinear bifurcations, from symmetry breaking to time-dependent modes, are investigated for the growth of an $8 \mathrm{~mm}$ diameter silicon crystal in a mono-ellipsoid mirror furnace. To build the confidence on our numerical simulation, some benchmarkings with previous studies and experiments are also considered. In the next section, the mathematical model and numerical solution are described. Section 3 is devoted to results and discussion, where detailed benchmark comparison will be also presented. Brief conclusions are drawn in Section 4.

\section{Model description and numerical solution}

A generic FZ crystal growth in a mono-ellipsoid mirror furnace is illustrated in Fig. 1a. Since axisymmetry is no longer assumed here, the system is described by a Cartesian coordinate $(x, y, z)$. The point-source model [3] for the mirror furnace is adopted for the heat input condition. This model is simple and only considers the primary light reflection from the point lamp source. Even though the higher-order reflections are ignored, the basic features of lamp heating still can be captured. The calculated superheating for a typical zone length of an $8 \mathrm{~mm}$ silicon rod being about $40^{\circ} \mathrm{C}$ is consistent with the detailed $2 \mathrm{D}$ simulation [1]. The sample pulling speed is $U_{\mathrm{c}}$, while the furnace upward speed $U_{h}$. For a pseudo-steady state growth, the furnace can be set at stationary $\left(U_{h}=0\right)$. The free surface is assumed rigid in this study due to the small free surface deformation in microgravity. The deformed meniscus will be

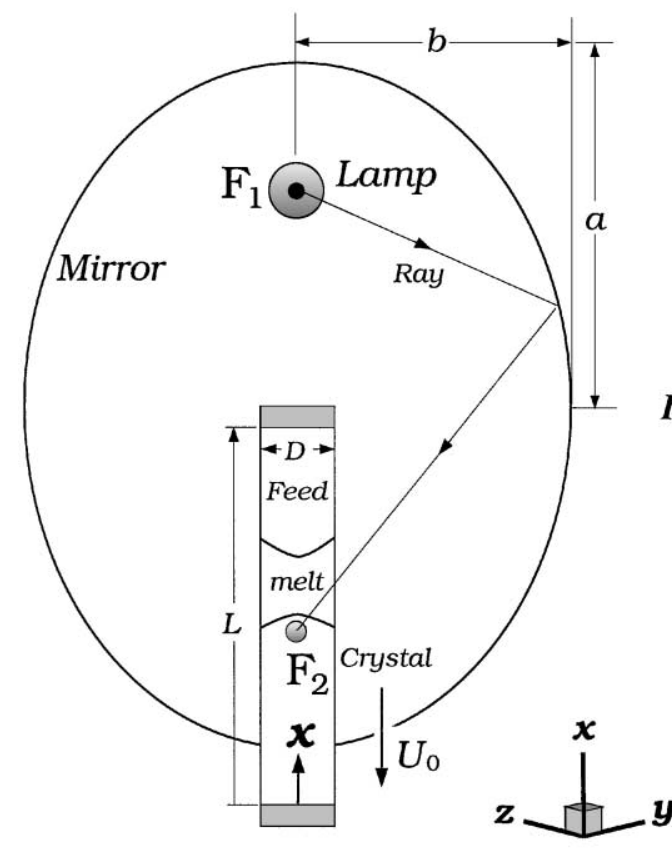

(a)

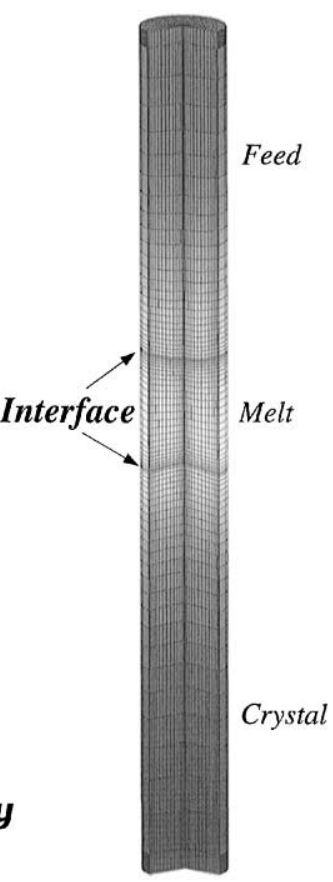

(b)

Fig. 1. (a) Schematic of floating-zone crystal growth in a mono-ellipsoid furnace; (b) a sample finite volume mesh (coarse grid) for calculation. 
considered in the near future. The flow and temperature fields as well as the melt/feed $\left(h_{\mathrm{f}}(x, y, z, t)\right)$ and melt/crystal $\left(h_{\mathrm{c}}(x, y, z, t)\right)$ interfaces are also represented in the Cartesian coordinate.

The dimensionless variables are defined by scaling length with the crystal diameter $D_{\mathrm{c}}$, time with $D_{\mathrm{c}}^{2} / \alpha_{\mathrm{m}}$, velocity with $\alpha_{\mathrm{m}} / D_{\mathrm{c}}$, pressure with $\rho_{\mathrm{m}} \alpha_{\mathrm{m}}^{2} / D_{\mathrm{c}}^{2}$, and temperature with the melting point $T_{\mathrm{m}}$, where $\alpha_{\mathrm{m}}$ is the thermal diffusivity and $\rho_{\mathrm{m}}$ the melt density. The time-dependent governing equations describing the convection and heat transport in the melt $(\mathrm{m})$ based on the Boussinesq approximation are as follows:

$\boldsymbol{\nabla} \cdot \mathbf{v}=0$

$\partial \mathbf{v} / \partial \tau+\mathbf{v} \cdot \boldsymbol{\nabla} \mathbf{v}=-\nabla P+\operatorname{Pr} \nabla^{2} \mathbf{v}$

$$
-\operatorname{Pr} \operatorname{Ra}_{\mathrm{T}}(\theta-1) \mathbf{e}_{\mathrm{g}},
$$

$\partial \theta / \partial \tau+\mathbf{v} \cdot \boldsymbol{\nabla} \theta=\nabla^{2} \theta$,

where $\tau, \mathbf{v}, P$, and $\theta$ are the dimensionless time, velocity, pressure, and temperature, respectively. Pr is the Prandtl number. In the source term of Eq. (2), $\mathbf{e}_{\mathrm{g}}$ is the unit vector of gravity, which is aligned with the growth axis in this study. Also, $T_{\mathrm{m}}$ serves as a reference temperature. The associated dimensionless number $\mathrm{Ra}_{T}$ is the thermal Rayleigh number, defined as follows:

$\mathrm{Ra}_{\mathrm{T}} \equiv \frac{g \beta_{\mathrm{T}} T_{\mathrm{m}} D_{\mathrm{c}}^{3}}{\alpha_{\mathrm{m}} v_{\mathrm{m}}}$

where $g$ is the gravitational acceleration, $\beta_{\mathrm{T}}$ the thermal expansion coefficients, and $v_{m}$ is the kinematic viscosity. In this study, due to the microgravity, $\mathrm{Ra}_{\mathrm{T}}=0$ is adopted.

In the crystal (c) and the feed (f) only heat transfer needs to be considered:

$$
\begin{aligned}
& \partial \theta / \partial \tau+\left(v_{i} \mathbf{e}_{z}+r \Omega_{i} \mathbf{e}_{\phi}\right) \cdot \boldsymbol{\nabla} \theta=\boldsymbol{\nabla} \cdot \tilde{\kappa}_{i} \boldsymbol{\nabla} \theta, \\
& \quad(i=\mathrm{c}, \mathrm{f}),
\end{aligned}
$$

where $r$ is the radial distance, $\Omega_{i}$ the dimensionless rotation speed, and $\tilde{\kappa}_{i} \equiv \alpha_{i} / \alpha_{\mathrm{m}}$ the dimensionless thermal diffusivity of feed and crystal; $\alpha_{i}$ is the thermal diffusivity of the feed $(i=\mathrm{f})$ or the crystal $(i=\mathrm{c})$. Also, $\mathbf{e}_{z}$ and $\mathbf{e}_{\phi}$ are the unit vectors in the axial and angular directions, respectively.
The no-slip condition is used for the velocity on solid boundaries:

$\mathbf{v}=\gamma_{\mathrm{c}}\left(v_{\mathrm{c}} \mathbf{e}_{z}+r \Omega_{i} \mathbf{e}_{\phi}\right)$,

where $\gamma_{\mathrm{c}} \equiv \rho_{\mathrm{c}} / \rho_{\mathrm{m}}$ and $v_{\mathrm{c}}$ is the dimensionless crystal pulling speed. At the free surface, the shear stress balance is imposed:

$\tau_{n s}=\mathrm{Ma}^{*} \partial \theta / \partial s$,

where $\tau_{n s}$ is the shear stress at the $n-s$ plane of the free surface; $\mathbf{n}$ and $\mathbf{s}$ are the unit normal and tangential vectors at the free surface, respectively. Also, $\mathrm{Ma}^{*}$ is the Marangoni number defined as

$\mathrm{Ma}^{*} \equiv \frac{(\partial \gamma / \partial T) T_{\mathrm{m}} D_{\mathrm{c}}}{\rho_{\mathrm{m}} v_{\mathrm{m}} \alpha_{\mathrm{m}}}$,

where $\partial \gamma / \partial T$ is the surface-tension-temperature coefficient of the melt. For better comparison with previous reports (e.g., [1] and [5]), two similar numbers are defined as the following:

$\mathrm{Ma} \equiv \mathrm{Ma}^{*} \Delta T L_{\mathrm{m}} /\left(2 T_{\mathrm{m}} D_{\mathrm{c}}\right) ; \quad \mathrm{Re} \equiv \mathrm{Ma} / \mathrm{Pr}$,

where $\Delta T$ is the maximum surface overheating $\left(T_{\max }-T_{\mathrm{m}}\right)$ and $L_{\mathrm{m}}$ is defined as the maximum zone length at the free surface. The Reynold's number $\mathrm{Re}$ is the same as the definition of $D_{\mathrm{c}} V / v_{\mathrm{m}}$, where $V \equiv(\partial \gamma / \partial T) \Delta T /\left(\rho_{\mathrm{m}} v_{\mathrm{m}}\right)$ is the characteristic thermocapillary velocity. In Eq. (6), two tangential directions need to be considered for the stress balance. Also, the kinematic condition at the free surface is also considered, i.e.,

$\mathbf{n} \cdot \mathbf{v}=0$,

where $\mathbf{n}$ is the unit normal vector at the free surface. Therefore, the velocity boundary condition at the free surface is then completed for its three components.

The thermal boundary conditions at the growth and feeding fronts are set by the heat flux balances:

$$
\begin{aligned}
& \left.Q\right|_{\mathrm{m}}-\left.Q\right|_{i}+\gamma_{\mathrm{c}}\left[\left(v_{i}+\mathrm{d} h_{i} / \mathrm{d} \tau\right) \mathbf{e}_{z}\right. \\
& \left.\quad-r \Omega_{i} \mathbf{e}_{\phi}\right] \mathrm{St} \cdot \mathbf{n}=0, \quad i=\mathrm{c} \text { or } \mathrm{f},
\end{aligned}
$$

where $\mathbf{n}$ is the unit normal vector at the feeding or growth interface pointing to the melt. $\left.Q\right|_{\mathrm{m}},\left.Q\right|_{\mathrm{c}}$, and $\left.Q\right|_{\mathrm{f}}$ are the dimensionless total heat fluxes at the melt, the crystal, and the feeding sides, respectively. The Stefan number $\mathrm{St} \equiv \Delta H /\left(C p_{\mathrm{m}} T_{\mathrm{m}}\right)$ scales the heat of fusion $(\Delta H)$ released during solidification to the sensible heat in the melt; $C p_{\mathrm{m}}$ 
is the specific heat of the melt. The component for $\left(r \Omega \mathbf{e}_{\phi} \cdot \mathbf{n}\right)$ is particular important for $3 \mathrm{D}$ interfaces during rotation. In this paper, the effects of rotation are not included due to the space limit. However, some interesting contribution of this term can be found elsewhere for similar applications [8].

The heat exchange between the sample and the furnace is by both convection and radiation according to the energy balance along the sample surface,

$$
\begin{aligned}
-\left.\mathbf{n} \cdot \kappa_{i} \boldsymbol{\nabla} \theta\right|_{i}= & \operatorname{Bi}\left(\theta-\theta_{\mathrm{amb}}\right)+\operatorname{Rad}_{i}\left(\theta^{4}-\theta_{\mathrm{amb}}^{4}\right) \\
& -\varepsilon_{\mathrm{L}} Q_{\mathrm{L}}, \quad(i=\mathrm{f}, \mathrm{m}, \mathrm{c})
\end{aligned}
$$

where $\mathbf{n}$ is the unit normal vector on the sample surface pointing outwards, $\mathrm{Bi} \equiv h D_{\mathrm{c}} / k_{\mathrm{m}}$ the Biot number, and $\operatorname{Rad}_{i} \equiv \sigma \varepsilon_{i} T_{\mathrm{m}}^{3} D_{\mathrm{c}} / k_{\mathrm{m}}$ the Radiation number, $h$ is the heat transfer coefficient, $k_{\mathrm{m}}$ the melt thermal conductivity, $\sigma$ the Stefan Boltzmann constant, and $\varepsilon_{i}$ the surface emissivity; $i=\mathrm{f}, \mathrm{c}, \mathrm{m}$, and $\mathrm{L}$ (the lamp). Temperature $\theta_{\mathrm{amb}}$ is the ambient temperature and $Q_{\mathrm{L}}$ is the dimensionless total heat flux from the lamp. Detailed calculation of $Q_{\mathrm{L}}$ can be found in our previous report [3]. A more realistic modeling of the lamp heating is possible (e.g., [1] and [9]), but it needs much more computing effort, which is less feasible for $3 \mathrm{D}$ simulation here. In the present study, all the boundary and heating conditions are axisymmetric. Therefore, any $3 \mathrm{D}$ results obtained here are due to the nonlinear bifurcation of the physical process.

The above governing equations and their associated boundary conditions can only be solved numerically. We have developed efficient FVM schemes using the primitive variable formulation and multigrid acceleration [10] for the free or moving boundary problem. For time-dependent calculations, a fully implicit Euler scheme is used for the time-derivative terms. A sample converged coarse-mesh for calculation is shown in Fig. $1 \mathrm{~b}$. As shown, finer grid spacing is placed near the interfaces to enhance the accuracy of calculation. Although the results obtained by the coarse mesh (the first level) are very close to that obtained by the fine mesh (the second level), in the following discussion, all the calculations are performed using the fine mesh, which contains eight times more of the finite volumes than that in Fig. 1b. A more detailed description of the numerical method can be found elsewhere $[10,11]$.

\section{Results and discussion}

Before presenting the results, we have performed extensive benchmark comparisons with previous works for both a full 2D model [3] and a simplified 3D model [5]. To save space, only the $3 \mathrm{D}$ comparison is presented here. For the simplified 3D model, the half-zone configuration is considered, where the upper and lower interfaces are assumed flat and fixed. For comparison purposes, the unit aspect ratio and $\operatorname{Pr}=0.01$ with an adiabatic free surface [5] are considered. Detailed physical properties used can be found in Table 1;

Table 1

Physical properties of silicon and some input parameters [3]

Physical Properties

$T_{\mathrm{m}}=1410^{\circ} \mathrm{C}$

$\Delta H=1803 \mathrm{~J} \mathrm{~g}^{-1}$

$k_{\mathrm{f}, \mathrm{c}}=0.22 T_{\mathrm{m}} / T \mathrm{~W} \mathrm{~cm}^{-1} \mathrm{~K}^{-1}$

$k_{m}=0.64 \mathrm{~W} \mathrm{~cm}^{-1} \mathrm{~K}^{-1}$

$C_{P_{\mathrm{f}, \mathrm{c}}}=1.038 \mathrm{~J} \mathrm{~g}^{-1} \mathrm{~K}^{-1}$

$C_{P_{\mathrm{m}}}=1.059 \mathrm{~J} \mathrm{~g}^{-1} \mathrm{~K}^{-1}$

$\partial \gamma / \partial T=-0.13 \mathrm{dyn} \mathrm{cm}^{-1} \mathrm{~K}^{-1}$

$\gamma=865+\left(T-T_{\mathrm{m}}\right) \partial \gamma / \partial T \mathrm{dyn} \mathrm{cm}^{-1}$

$\mu_{\mathrm{m}}=0.007 \mathrm{~g} \mathrm{~cm}^{-1} \mathrm{~s}^{-1}$

$\beta_{T}=1.32 \times 10^{-4} \mathrm{~K}^{-1}$

$\varepsilon_{\mathrm{f}, \mathrm{c}}=0.7$

$\varepsilon_{\mathrm{m}}=0.3$

$\rho_{s}=2.33 \mathrm{~g} \mathrm{~cm}^{-3}$

$\rho_{\mathrm{m}}=2.55 \mathrm{~g} \mathrm{~cm}^{-3}$

Input parameters

$D_{\mathrm{f}, \mathrm{c}}=0.8 \mathrm{~cm}$

$U_{\mathrm{f}, \mathrm{c}}=0--24 \mathrm{~cm} \mathrm{~h}^{-1}$

$U_{h}=0 \mathrm{~cm} \mathrm{~h}^{-1}$

$\Omega_{\mathrm{f}, \mathrm{c}}=0 \mathrm{RPM}$

$L=8 \mathrm{~cm}$

$T_{\mathrm{amb}}=200^{\circ} \mathrm{C}$

$a=9 \mathrm{~cm}$

$b=8 \mathrm{~cm}$

$W_{\mathrm{L}}=700 \mathrm{~W}$

$\varepsilon_{\mathrm{L}}=0.8$

$h=1.1 \times 10^{-3} \mathrm{~W} \mathrm{~cm}^{-2} \mathrm{~K}^{-1}$ 
however, for the half zone, the viscosity is adjusted slightly to match $\operatorname{Pr}=0.01$. The calculated bifurcation diagram for the maximum surface temperature difference (in the angular direction) at the mid-height is illustrated in Fig. 2; the absolute value of the Re number is used. As shown, the primary bifurcation is supercritical and bifurcates from an axisymmetric mode or the so-called $m 0$ mode (like (a) in Fig. 2) to a 3D two-fold $(\mathrm{m} 2)$ mode (such as (b)) at $\operatorname{Re}_{\mathrm{cl}} \approx 1980$, while the secondary bifurcation or the Hopf point (at $\mathrm{Re}_{\mathrm{c} 2} \approx 7400$ ) is to a time-dependent $m 2$ mode. This result is in good agreement with previous calculations $\left(\operatorname{Re}_{\mathrm{c} 1}=1960\right.$ and $\operatorname{Re}_{\mathrm{c} 2}=6250$ in Ref. [5] by a finite element method, $\operatorname{Re}_{\mathrm{c} 1}=2020$ and $\mathrm{Re}_{\mathrm{c} 2}=6880$ in Ref. [12] by a finite difference method, and $\mathrm{Re}_{\mathrm{c} 1}=1890$ in Ref. [6] by the linear stability analysis). Also, at $\operatorname{Re}=3500$ (see (b) in Fig. 2$)$, the calculated maximum velocities $\left(u_{\max }=\right.$ 0.08716 and $\left.v_{\phi}=0.01476\right)$ also agree well with previous calculation $\left(u_{\max }=0.08607\right.$ and $v_{\phi}=$ 0.01340 in Ref. [5]).

At $\mathrm{Re}=2 \times 10^{4}$, the periodic oscillations of four surface temperatures $\left(90^{\circ}\right.$ apart at the middle surface) are also shown in Fig. 2, where some flow and isotherms of a period of oscillation are also illustrated in Fig. 3. As shown, the dimensionless frequency is about one and the isotherms are not rotating; there is no exchange of the high and low temperatures in the angular direction. This flow structure moving back-and-forth is also consistent with the previous calculation [5]. However, we find that the periodic and non-rotating oscillation is retained only up to $\mathrm{Re} \approx 4 \times 10^{4}$. Beyond that, there is a wave motion in the azimuthal angle direction and it travels slowly. As a result, side (lower) frequencies appear, and this new bifurcation point may be denoted by $\mathrm{Re}_{\mathrm{c} 3}$. Nevertheless, at $\mathrm{Re}=5 \times 10^{4}$, the angular speed is changing with time, and sometimes the wave direction can be reversed. As will be discussed shortly, this angular wave may be related to the significant back melting during crystal growth. Further comparison is also conducted for the half-zone experiment by Nakamura et al. [7] in microgravity; the zone length $=1 \mathrm{~cm}, D_{\mathrm{c}}=1 \mathrm{~cm}$, and $\Delta T=150^{\circ} \mathrm{C}$. The calculated heat flows also show some wave structure in the angular direction. Interestingly,

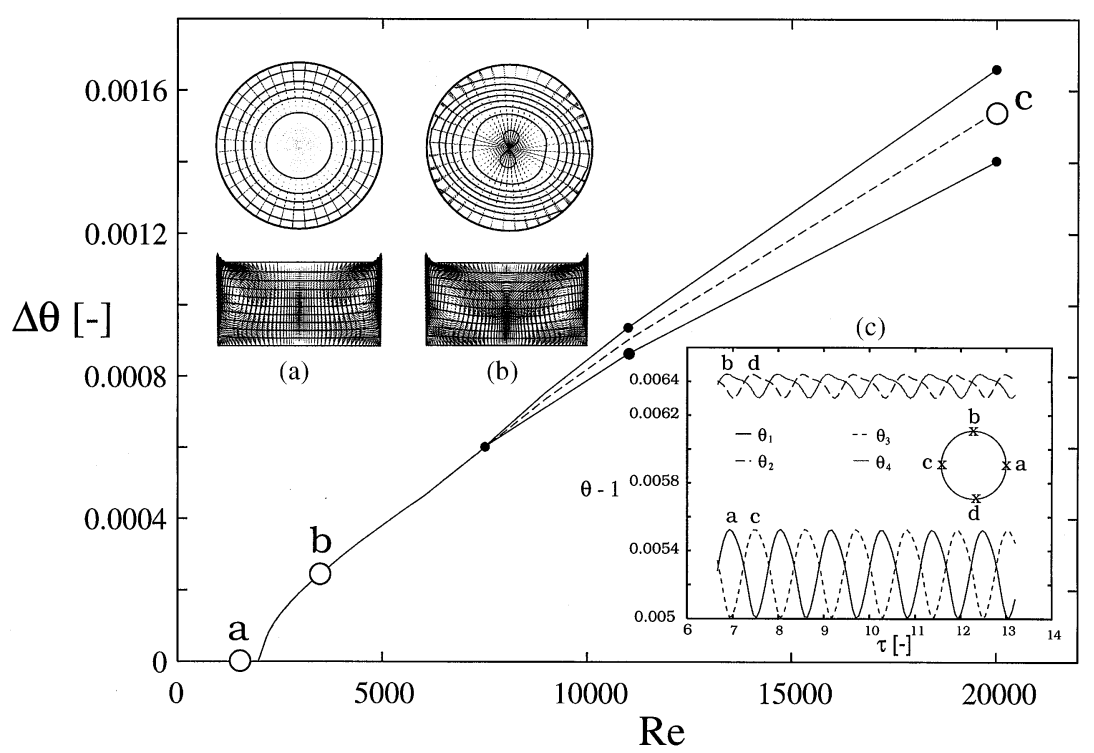

Fig. 2. Bifurcation diagram for the half-zone configuration (aspect ratio=1 and $\mathrm{Pr}=0.01$ ). (a) Flow structure and isotherms at $\mathrm{Re}=1500$; (b) flow structure and isotherms at $\mathrm{Re}=3500$; (c) oscillation of four surface temperatures at the mid-height for $\mathrm{Re}=2 \times 10^{4}$. After the Hopf point, the solid lines indicate the mean values of the high and low temperatures during oscillation. 


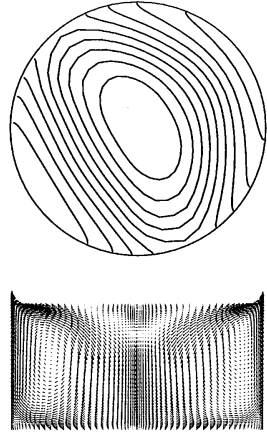

(a)
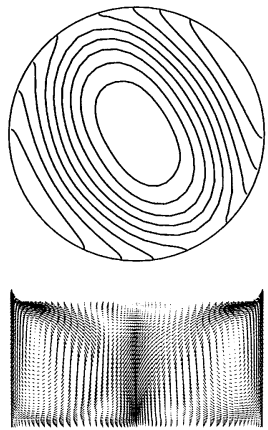

(d)
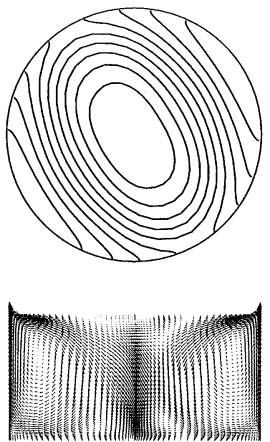

(b)
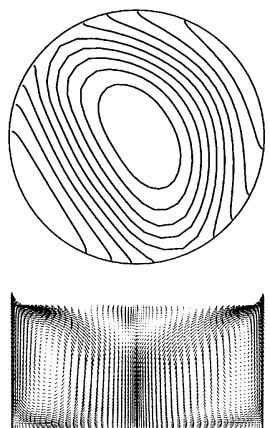

(e)

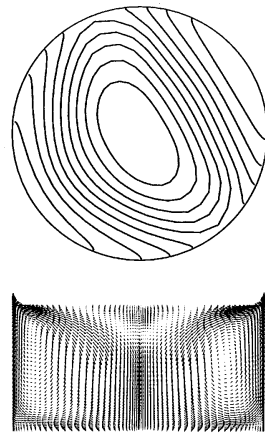

(c)

Fig. 3. Some flow patterns and isotherms at $\mathrm{Re}=2 \times 10^{4}$ for (c) in Fig. 2. The time interval is about 0.25 (dimensionless).

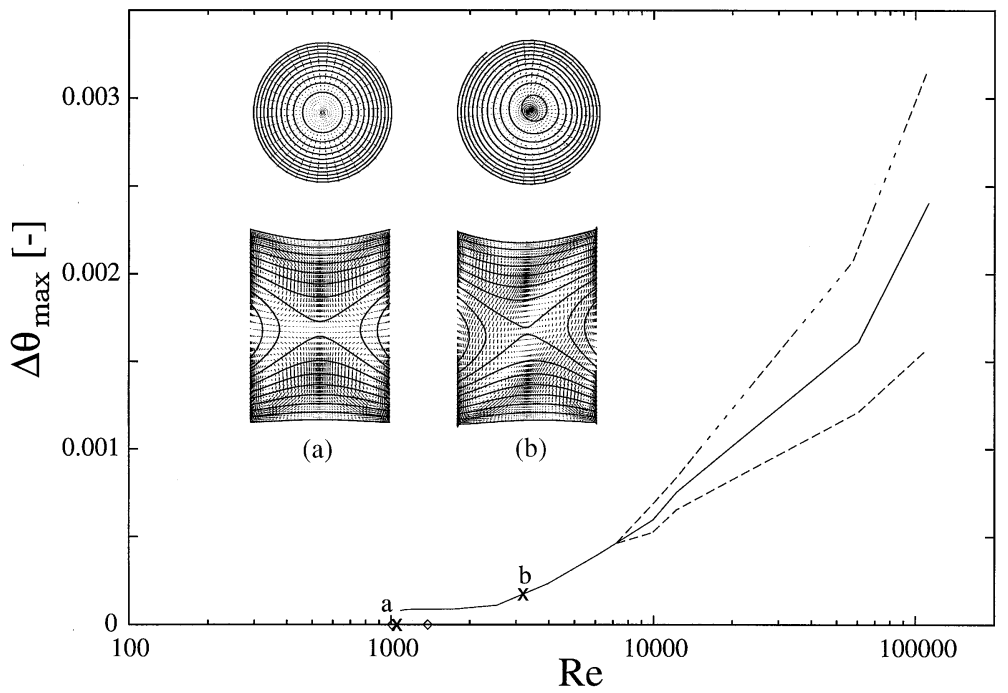

Fig. 4. Bifurcation diagram for the full-zone configuration with moving interfaces; (a) an axisymmetric solution $(m 0)$ at Re $=1083$; (b) a $3 \mathrm{D} m 1$ solution at $\mathrm{Re}=3200$. The lamp power is $700 \mathrm{~W}$. 
the fast Fourier analysis of the temperature oscillation ( $2 \mathrm{~mm}$ above the lower boundary) agrees very well with the measured one; the frequency is about $0.1-0.2 \mathrm{~Hz}$. In addition, the calculated amplitudes (about $20^{\circ} \mathrm{C}$ ) of the temperature oscillation are also consistent with the experiments.

For full zone calculations, the growth of an $8 \mathrm{~mm} \mathrm{Si}$ crystal in microgravity is considered; the heating configuration is as that illustrated in Fig. 1a and the power is set at $700 \mathrm{~W}$. Again, the detailed physical properties and input conditions are listed in Table 1. The calculated bifurcation diagram is summarized in Fig. 4, where the Re number is adjusted by changing $\partial \gamma / \partial T$; the maximum temperature difference and zone length are taken after the calculation is finished. Similar to the half-zone configuration, as shown in Fig. 4, there are also two typical bifurcation points. The primary bifurcation, however, is from an $m 0$ to an $m 1$ mode. Interestingly, it is subcritical; there is a hystersis at $\mathrm{Re} \approx 1000$. Even with the coarse grid, the subcriticality is obvious. Although, this point is far away from the typical growth condition $(\mathrm{Re} \approx$ $10^{5}$ ), it is again a good example showing the coupling of the flow bifurcation and the deformable interfaces. Similar bifurcation for the buoyancy convection in the vertical zone-melting was also observed by Lan and Liang [11]. The range of this hysteresis also agrees reasonably well with the reported values (ranging from $\mathrm{Re} \approx 1000$ to 1500). The time-dependent mode appear at $\operatorname{Re} \approx 7000$, and the $m 1$ flow structure is dominant. Again, these are consistent with previous reports $[1,2,5]$. Because of the low Prandtl number, the deformation of the interfaces is not much and deviates only slightly from the axisymmetry $(m 0)$, as shown by (b) in Fig. 4.

At $\operatorname{Re}=1.1 \times 10^{5}$ by using a typical value of $\partial \gamma / \partial T\left(-0.13 \mathrm{dyn} / \mathrm{cm}^{-1} \mathrm{~K}^{-1}\right)$, the flow oscillation becomes much more severe. The most interesting part is the induced oscillation of the interface speed. As shown in Fig. 5a, the oscillation ampltude is comparable to the growth rate (2-3 $\left.\mathrm{mm} \mathrm{min}^{-1}\right)$; four interface speeds $\left(90^{\circ}\right.$ apart) at the surface are monitored. As shown, significant back melting with frequency in $0.1-0.4 \mathrm{~Hz}$ is obvious. The significant back melting was also observed in a recent experiment [2], which was performed using an 8-10 $\mathrm{mm}$ diameter silicon rod with a much longer zone (about $16 \mathrm{~mm}$ ). The result of the fast Fourier transform of the growth rates is shown in Fig. 5b; four positions with $90^{\circ}$ apart are considered. Clearly, the low frequency mode at about $0.1 \mathrm{~Hz}$ seems to be more significant. However, it should be pointed out that, this mode corresponding to the significant growth and back melting may be due to the angular thermal waves. Due to the larger values, their Fourier's components are also larger. Meanwhile, the high frequency mode, which corresponds to the local flow oscillation, has smaller oscillation amplitudes, and hence the Fourier's components are smaller.
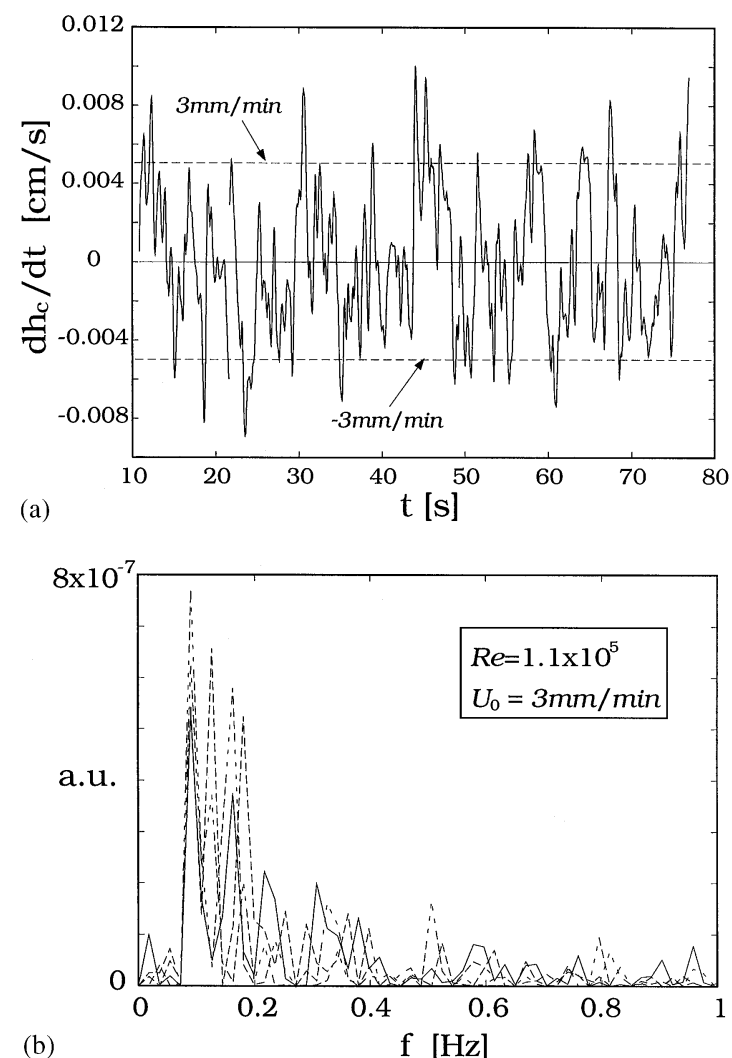

Fig. 5. (a) Calculated interface speed at $\operatorname{Re}=1.1 \times 10^{5}$. The dash lines indicate the typical growth and melting velocity at $3 \mathrm{~mm} / \mathrm{l}$; (b) power spectrum of four interface speeds at the free surface. 
The mean value of the growth rate from the view of the observer is zero here. One can further compare the time domain oscillations shown in Fig. 5a and their corresponding Fourier's components in Fig. $5 b$ to get a better picture.

If we take the some flow patterns and isotherms at the three cross sections (at $y=0, z=0$, and $x=$ 0.55), as shown in Fig. 6, the nature of interface oscillations becomes clearer; the time interval is 1s in Fig. 6. As illustrated in Fig. 6, the angular motion of the isotherms, though not very regular, is obvious. This is also similar to the half-zone configuration at $\operatorname{Re}=5 \times 10^{4}$, even through it has an $m 2$ flow structure. The back melting occurs when the high temperature front arrives; by the time the zone length there also becomes longer. Meanwhile, as the cold front comes, the place having the lowest surface temperature, or the shortest zone length, grows faster. This simulation result thus provides a clear picture to the interesting back melting observed in experiments [2].

\section{Conclusions}

A self-consistent $3 \mathrm{D}$ model considering heat flows and interfaces is developed for the FZ silicon growth in a mirror furnace. The calculated results for the half-zone configuration agrees well with previous calculations and experiments. The bifurcation behavior of the growth of an $8 \mathrm{~mm}$ silicon crystal in a mono-ellipsoid mirror furnace is also investigated. It is found that the primary bifurcation is subcritical, and the $3 \mathrm{D} m 1$ modes are the dominant flow structures. For the case corresponding to a real growth situation, the angular traveling wave leads to significant back melting along the rim of the growth interface, which is consistent with the experimental observation. However, the speed of the traveling wave is not constant. In the present model, the free surface is assumed to be flat and rigid. Although we do not anticipate any significant effect from the deformable free surface, the release of this assumption and

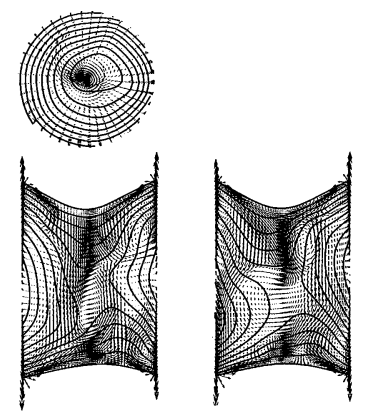

(a)

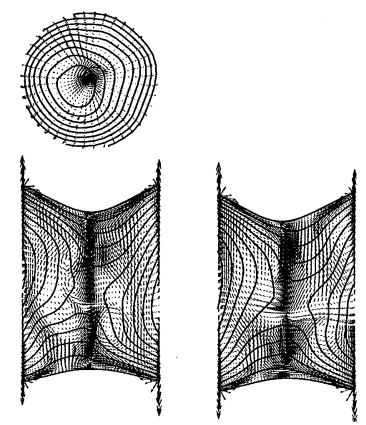

(d)
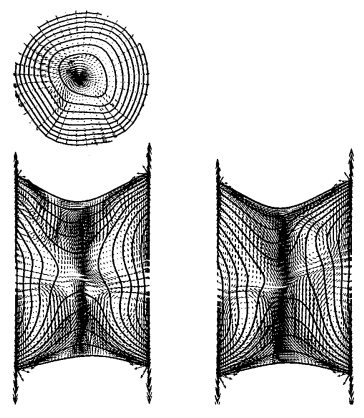

(b)
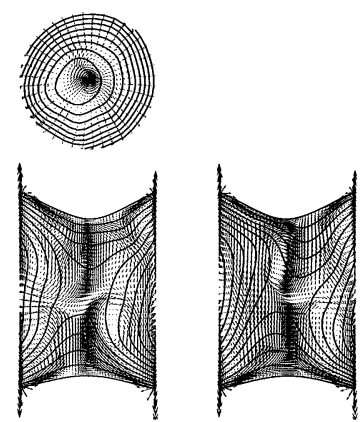

(e)

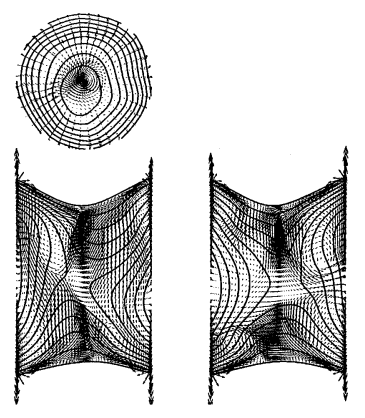

(c)

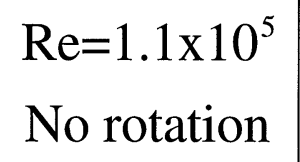

Fig. 6. Some flow patterns and isotherms at $\mathrm{Re}=1.1 \times 10^{5}$; time interval is about $1 \mathrm{~s}$ and the spacing for isotherms is $2.9^{\circ} \mathrm{C}$. 
the coupling of the free surface and Marangoni instability will be considered in the near future.

\section{Acknowledgements}

The authors are grateful for the support from the National Science Council and the National Center for High Performance Computing of the Republic of China under Grant No. NSC89-2214E002-040.

\section{References}

[1] P. Dold, A. Croll, K.W. Benz, J. Crystal Growth 183 (1998) 545.

[2] M. Schweizer, A. Croll, P. Dold, Th. Kaiser, M. Lichtensteiger, K.W. Benz, J. Crystal Growth 203 (1999) 500.
[3] C.W. Lan, J. Crystal Growth 169 (1996) 269.

[4] A. Muhlbauer, A. Muiznieks, G. Raming, H. Riemann, A. Ludke, J. Crystal Growth 198/199 (1999) 107.

[5] M. Levenstam, G. Amberg, J. Fluid Mech. 297 (1995) 357.

[6] M. Prange, M. Wanschura, H.C. Huhlmann, H.J. Rath, J. Fluid Mech. 394 (1999) 281.

[7] S. Nakamura, T. Hibiya, K. Kakimoto, N. Imaishi, S. Nishizawa, A. Hirata, K. Mukai, S. Yoda, T.S. Morita, J. Crystal Growth 186 (1998) 85.

[8] C.W. Lan, C.H. Chian, T.Y. Wang, J. Crystal Growth 218 (2000) 115.

[9] C.W. Lan, C.H. Tsai, J. Crystal Growth 173 (1997) 561.

[10] C.W. Lan, M.C. Liang, J. Comp. Phys. 152 (1999) 55.

[11] C.W. Lan, M.C. Liang, J. Crystal Growth 208 (2000) 327.

[12] N. Imaishi, private communication. 